\title{
Comparison of Off-line IR Bump and Action-Angle Kick Minimization
}

\author{
Y. Luo, F. Pilat, V. Ptitsyn, D. Trbojevic, J. Wei
}

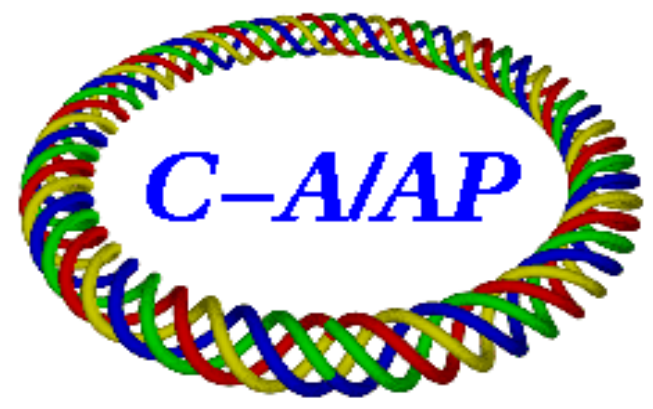

Collider-Accelerator Department Brookhaven National Laboratory Upton, NY 11973 


\title{
Comparison of Off-line IR Bump and Action-Angle Kick Minimization*
}

\author{
Y. Luo, F. Pilat, V. Ptitsyn, D. Trbojevic, J. Wei \\ Brookhaven National Laboratory, Upton, NY 11973
}

\section{Abstract}

The Interaction region bump (IR bump) nonlinear correction method has been used for the sextupole and octupole field error on-line corrections in the Relativistic Heavy Ion Collider (RHIC) [1, 2]. Some differences were found for the sextupole and octupole corrector strengths between the on-line IR bump and the predictions from the action-angle kick minimization [3]. In this report we comparise the corrector strengths from the off-line IR bump correction and the action-angle kick minimization based on the RHIC Blue ring lattice with the IR nonlinear modeling [4]. The comparison confirms that there are some differences between resulting corrector strengths. After an item-by-item check, the source of the difference between two methods is found and discussed. It is followed by further discussion of the operational IR bump applications to the octupole, and skew sextupole and skew quadrupole field error corrections.

\section{ACTION-ANGLE KICK MINIMIZATION}

\section{Principle}

There are several ways to correct the local nonlinear field errors located in the interaction regions [5]. The actionangle kick minimization is a fast and simple one. It has been used for the Large Hadron Collider (LHC) and RHIC dead-reckoning corrections for the interaction region nonlinear field errors. Using the action and angle canonical variables in the framework of Hamiltonian mechanism, this method minimizes the action change due to the nonlinear field errors in the interaction region for the round beams, order by order.

To minimize the action change for each order is equivalent to minimize the following two quantities simultaneously [3]:

$$
\oint_{L} d s C_{z} c_{n}+(-1)^{n+1} \oint_{R} d s C_{z} c_{n},
$$

where $L$ and $R$ mean the left and right sides of the interaction region, $z$ stands for $x$ or $y$ plane, $c_{n}$ stands for the normal or skew field errors $b_{n}$ or $a_{n}, n$ is the field error order. $C_{z}$ is the weight factor, which is different for different order of errors and for different $x$ or $y$ plane. The integrations or summations are taken across the interaction region.

The American magnetic field order convention is used, where the $n=0$ term represents the dipole field. The correction weight factors are given according to the multipoles

\footnotetext{
*Work supported by U.S. DOE under contract No DE-AC02$98 \mathrm{CH} 10886$
}

as:

$$
\begin{aligned}
C_{x} & = \begin{cases}\beta_{x}^{(n+1) / 2} & \text { for } b_{n} \\
\beta_{x}^{n / 2} \beta_{y}^{1 / 2} & \text { for } a_{n}\end{cases} \\
C_{y} & = \begin{cases}\beta_{y}^{(n+1) / 2} & \text { for odd } b_{n} \text { and even } a_{n} \\
\beta_{x}^{1 / 2} \beta_{y}^{n / 2} & \text { for even } b_{n} \text { and odd } a_{n}\end{cases}
\end{aligned}
$$

For each order, there are two quantities to be minimized, one in the horizontal plane, one in the vertical plane. Therefore at least two nonlinear correctors are needed for each order correction. A natural choice is to place the correctors at either side of the interaction region.

In order to facilitate the following discussion, Table 1 lists the weight factors for different field error $b_{n}$ and $a_{n}$.

Table 1: Weight factors for action-angle kick minimization

\begin{tabular}{|c|c|c|c|c|}
\hline Order & $C_{x}$ & $C_{y}$ & $\begin{array}{c}k_{n} l \\
/ k_{n s} l\end{array}$ & $(-1)^{n+1}$ \\
\hline$b_{2}$ & $\beta_{x}^{3 / 2}$ & $\beta_{x}^{1 / 2} \beta_{y}$ & $k_{2} l$ & -1 \\
\hline$b_{3}$ & $\beta_{x}^{2}$ & $\beta_{y}^{2}$ & $k_{3} l$ & 1 \\
\hline$b_{4}$ & $\beta_{x}^{5 / 2}$ & $\beta_{x}^{1 / 2} \beta_{y}^{2}$ & $k_{4} l$ & -1 \\
\hline$b_{5}$ & $\beta_{x}^{3}$ & $\beta_{y}^{3}$ & $k_{5} l$ & 1 \\
\hline$a_{2}$ & $\beta_{x} \beta_{y}^{1 / 2}$ & $\beta_{y}^{3 / 2}$ & $k_{2 s} l$ & -1 \\
\hline$a_{3}$ & $\beta_{x}^{3 / 2} \beta_{y}^{1 / 2}$ & $\beta_{x}^{1 / 2} \beta_{y}^{3 / 2}$ & $k_{3 s} l$ & 1 \\
\hline$a_{4}$ & $\beta_{x}^{2} \beta_{y}^{1 / 2}$ & $\beta_{y}^{5 / 2}$ & $k_{4 s} l$ & -1 \\
\hline$a_{5}$ & $\beta_{x}^{5 / 2} \beta_{y}^{1 / 2}$ & $\beta_{x}^{1 / 2} \beta_{y}^{5 / 2}$ & $k_{5 s} l$ & 1 \\
\hline
\end{tabular}

\section{Discussion}

The action-angle kick minimization assumes that the phase advances in the $x$ and $y$ planes across the interaction point are close to $\pi$. This condition is always satisfied for most colliders' interaction regions, where small $\beta^{\star}$ functions are used to achieve high colliding luminosities. The phase advances in the triplet on each side are very small. The $\pi$ phase jump happens in the region around the interaction point (Fig. 2). The RHIC interaction region includes separation dipole magnets, triplet quadrupoles and nonlinear field correction packages.

Global nonlinear correction schemes (like the resonance driving term correction) also work for the local error compensation where the error sources and correctors are located in the same region. Eq. 1 is derived from the minimization of the local action change in the interaction region. It also can be derived in other ways, such as minimizing the global Hamiltonian resonance driving strength of one specific order in the interaction region. 
It should be pointed out that the weight factors for different order of the field errors in Eq. 1 only come from the highest order of the resonances in the horizontal and vertical planes, since round beams are assumed.

For example, the sextupoles could introduce $Q_{x}=p$, $3 Q_{x}=p, Q_{x} \pm 2 Q_{y}=p$ resonances. To fully correct all the resonances, we should minimize all the following resonance strengths: [6]

$$
\left\{\begin{array}{ccc}
\sum_{j} k_{2} l \beta_{x}^{1 / 2} \beta_{y} e^{i \Psi_{x}} & \longrightarrow 0 \\
\sum_{j} k_{2} l \beta_{x}^{3 / 2} e^{i \Psi_{x}} & \longrightarrow 0 \\
\sum_{j} k_{2} l \beta_{x}^{3 / 2} e^{i 3 \Psi_{x}} & \longrightarrow 0 \\
\sum_{j} k_{2} l \beta_{x}^{1 / 2} \beta_{y} e^{i\left(\Psi_{x}-2 \Psi_{y}\right)} & \longrightarrow 0 \\
\sum_{j} k_{2} l \beta_{x}^{1 / 2} \beta_{y} e^{i\left(\Psi_{x}+2 \Psi_{y}\right)} & \longrightarrow 0
\end{array}\right.
$$

where $k_{2} l$ is the sextupole integrated strength. The actionangle kick minimization assumes that the beam is round, so it does corrections of the highest order resonances $3 Q_{x}=p$ and $Q_{x}+2 Q_{y}=p$ in the $x$ and $y$ planes, respectively. The weight factors for them are $\beta_{x}^{3 / 2}$ and $\beta_{x}^{1 / 2} \beta_{y}$, respectively.

Octupoles induce $4 Q_{x}=p, 4 Q_{y}=p, 2 Q_{x}=p$, $2 Q_{y}=p, 2 Q_{x} \pm 2 Q_{y}=p$ resonances. To correct all the resonances, we should minimize all the following resonance strengths:

$$
\left\{\begin{array}{ccc}
\sum_{j} k_{3} l \beta_{x}^{2} e^{i 4 \Psi_{x}} & \longrightarrow 0 \\
\sum_{j} k_{3} l \beta_{y}^{2} e^{i 4 \Psi_{y}} & \longrightarrow 0 \\
\sum_{j} k_{3} l \beta_{x} \beta_{y} e^{i 2 \Psi_{x}} & \longrightarrow 0 \\
\sum_{j} k_{3} l \beta_{x}^{2} e^{i 2 \Psi_{x}} & \longrightarrow 0 \\
\sum_{j} k_{3} l \beta_{x} \beta_{y} e^{i 2 \Psi_{y}} & \longrightarrow & 0 \\
\sum_{j} k_{3} l \beta_{y}^{2} e^{i 2 \Psi_{y}} & \longrightarrow & 0 \\
\sum_{j} k_{3} l \beta_{x} \beta_{y} e^{i\left(2 \Psi_{x}+2 \Psi_{y}\right)} & \longrightarrow & 0 \\
\sum_{j} k_{3} l \beta_{x} \beta_{y} e^{i\left(2 \Psi_{x}-2 \Psi_{y}\right)} & \longrightarrow & 0
\end{array}\right.
$$

where $k_{3} l$ is the octupole integrated strength. The Actionangle kick minimization assumes that the beam is round, so it corrects the leading resonances $4 Q_{x}=p$ and $4 Q_{y}=p$ in $x$ and $y$ planes, respectively. Their correction weight factors are $\beta_{x}^{2}$ and $\beta_{y}^{2}$, respectively.

\section{IR BUMP CORRECTION}

The IR bump correction method is an elegant way for operational IR nonlinear corrections in a real machine. It creates a local horizontal or vertical orbit bump across the interaction region to simulate the resonance corrections. Small tune shifts due to the bump are measured with a high resolution phase lock loop( PLL ) system. Since the relations between the tune shifts and the bump amplitudes are different for different orders of the nonlinear field errors, the IR bump correction is performed order by order by minimizing the polynomial fitting terms of the tune shifts.

For the on-line RHIC IR bump, the bump amplitude changes in a continuous way. The different order polynomial terms of the tune shifts with the bump amplitudes are obtained through the polynomial fitting. For example, the linear terms of the polynomial fitting in the $x$ plane IR bump are related to the sextupole field errors. The quadratic term of the polynomial fitting in the $x$ plane IR bump are related to the octupole field error.

In the procedures of the sextupole and octupole IR bump corrections, we use two sextupole correctors to minimize the linear terms of the $x$ and $y$ tune shifts to zero simultaneously and use two octupole correctors to minimize the quadratic terms of the $x$ and $y$ tune shifts to zero simultaneously. In the following, we show the analytical comparisons of the IR bump correction and the action-angle minimization methods for the sextupole and octupole nonlinear corrections.

\section{Sextupole Error Correction}

The tune shifts from the sextupoles with the horizontal beam orbit offsets are given by:

$$
\left\{\begin{array}{l}
\Delta Q_{x}=\frac{1}{2 \pi} \sum\left(6 b_{2} x_{c o} \beta_{x}\right) d s \\
\Delta Q_{y}=\frac{1}{2 \pi} \sum\left(-6 b_{2} x_{c o} \beta_{y}\right) d s
\end{array}\right.
$$

Considering the phase advance $\pi$ on the interaction region, one concludes that the local horizontal orbit bump is proportional to the $\beta_{x}^{1 / 2}$,

$$
\left\{\begin{array}{ccc}
x_{c o} & \propto & \beta_{x}^{1 / 2}, \text { in one side of IR } \\
x_{c o} & \propto & -\beta_{x}^{1 / 2}, \text { in another side of IR }
\end{array} .\right.
$$

For simplicity, in the following analysis and simulation we assume that the orbit at one point in the IR bump is proportional to the bump step number. The orbit bump at different bump steps are given by:

$$
x_{c o}=x_{c o, 1} \times k,
$$

$x_{c o, 1}$ is the local orbit bump amplitude step, $k=$ $-N,-(N-1), \ldots,(N-1), N . N$ is the maximum bump step No..

The total tune shifts due to the $x$ plane IR bump are:

$$
\left\{\begin{array}{ccc}
\Delta Q_{x} & \propto & \left(\sum_{L} b_{2} d s \beta_{x}^{3 / 2}-\sum_{R} b_{2} d s \beta_{x}^{3 / 2}\right) \cdot k \\
\Delta Q_{y} & \propto \quad\left(\sum_{L} b_{2} d s \beta_{x}^{1 / 2} \beta_{y}-\sum_{R} b_{2} d s \beta_{x}^{1 / 2} \beta_{y}\right) \cdot k
\end{array} .\right.
$$

If we use the sextupole correctors in the interaction region to minimize the linear polynomial terms of the tune shifts with the bump steps $k$, we obtain:

$$
\left\{\begin{array}{ccc}
\sum_{L} b_{2} d s \beta_{x}^{3 / 2}-\sum_{R} b_{2} d s \beta_{x}^{3 / 2} & = & 0 \\
\sum_{L} b_{2} d s \beta_{x}^{1 / 2} \beta_{y}-\sum_{R} b_{2} d s \beta_{x}^{1 / 2} \beta_{y} & = & 0
\end{array},\right.
$$

then we think that the sextupole field errors in this interaction region is corrected.

Eq. 10 is the same equation to what one obtains from the action-angle kick minimization for the sextupole field error correction, using Eq. 1 and Table 1. Up to now, there is no difference between the IR bump correction method and the action-angle kick minimization method. 


\section{Octupole Error Correction}

For the octupole IR bump correction, we also use the $x$ plane bump, the tune shifts from the octupoles due to the orbit bump are:

$$
\left\{\begin{array}{l}
\Delta Q_{x}=\frac{1}{2 \pi} \sum\left(12 b_{3} x_{c o}^{2} \beta_{x}\right) d s \\
\Delta Q_{y}=\frac{1}{2 \pi} \sum\left(-12 b_{3} x_{c o}^{2} \beta_{y}\right) d s .
\end{array}\right.
$$

Substituting Eq.7 and Eq. 8 into the above equation, we obtain the tune shifts due to the IR bump at every bump step $k$ :

$$
\left\{\begin{array}{ccc}
\Delta Q_{x} & \propto\left(\sum_{L} b_{3} d s \beta_{x}^{2}+\sum_{R} b_{3} d s \beta_{x}^{2}\right) \cdot k^{2} \\
\Delta Q_{y} & \propto\left(\sum_{L} b_{3} d s \beta_{x} \beta_{y}+\sum_{R} b_{3} d s \beta_{x} \beta_{y}\right) \cdot k^{2}
\end{array} .\right.
$$

Using the octupole correctors in the interaction region to minimize the quadratic terms of the tune shifts with the bump steps $k$, we obtain:

$$
\left\{\begin{array}{ccc}
\sum_{L} b_{3} d s \beta_{x}^{2}+\sum_{R} b_{3} d s \beta_{x}^{2} & = & 0 \\
\sum_{L} b_{3} d s \beta_{x} \beta_{y}+\sum_{R} b_{3} d s \beta_{x} \beta_{y} & = & 0
\end{array} .\right.
$$

This equation for the $x$ plane is the same to what we get from the action-angle kick minimization for the octupole correction from Table 1. The weight factor $\beta_{x} \beta_{y}$ in the second equation for the vertical plane is different from $\beta_{y}^{2}$ from Table 1. We will come back to discuss this issue later.

\section{IR Bump Setup}

There are several strict demands to setup the IR bump. First, the IR bump correction assumes that the IR bump should be localized in the interested interaction region. The measured tune shifts should solely come from the nonlinear fields in this region. IR bump closure is therefore an important issue.

The orbit trajectory produced by the IR bump has to emulate exactly a free betatron oscillation through the whole region where errors and correctors are located. Thus the orbit through that region is described by:

$$
x_{c o}=A \sqrt{\beta} \sin \left(\Psi_{x}+\psi_{0}\right),
$$

where $A$ and $\psi_{0}$ are constant. This only holds between two dipole kickers. If one uses three dipole kicker bump, the interaction region errors and the nonlinear correctors should be all located between two of the three dipole kickers.

Since the phase advance on the interaction region is close to $\pi$, the orbit produced by the IR bump is positive on one side and negative on the other side of the interaction region. The local orbit excursion in the IR bump is proportional to the local $\beta^{1 / 2}$.

\section{IR BUMP SIMULATION}

The MADX code [7] is used to simulate the IR bump correction. At each bump step, we set the right dipole kicker strengths to produce the certain orbit bump amplitude. MADX calculates the tune shifts at each bump step.
By fitting the tune shifts with the bump steps, the $k$ in Eq. 8 , not the bump amplitudes, the different orders of the polynomial terms are obtained.

For simplicity, in the following we only focus on the IR bump correction simulation in the 8 o'clock interaction region in the RHIC Blue ring. The dipole kickers are the same as those used in the on-line application. The three horizontal dipole kickers bo7-th4, bi8-th3, bi8-th5 are used to create the horizontal IR bump. The interaction region, including the two DX and two D0 dipole magnets, six triplet quadrupoles and the IR nonlinear correction packages are all located between bo7-th4 and bi8-th3.

Fig. 1 shows the optics and the magnet locations in the 8 o'clock interaction region of the RHIC Blue ring. Fig. 2 shows the betatron phase across the interaction region. The $\beta$ function at the IP is $1 \mathrm{~m}$. Figure 3 shows an example of the orbit with the horizontal IR bump at 8 o'clock interaction region. The orbit leakage due to the IR bump is seen in Figure 3. However, the bump closure is still acceptable. In the simulation, the maximum orbit amplitude in the IR bump is chosen below $5 \mathrm{~mm}$.

Fig. 4 gives one example of the tune shifts from the simulation. The tune shifts are obtained with respect to that with zero bump amplitude, or the 11th bump step in the simulation. Up to seven order polynomial fittings are performed, the fitting curve is also plotted in Fig. 4. Fig.5 shows the tune shift contributions from the first three polynomial terms.

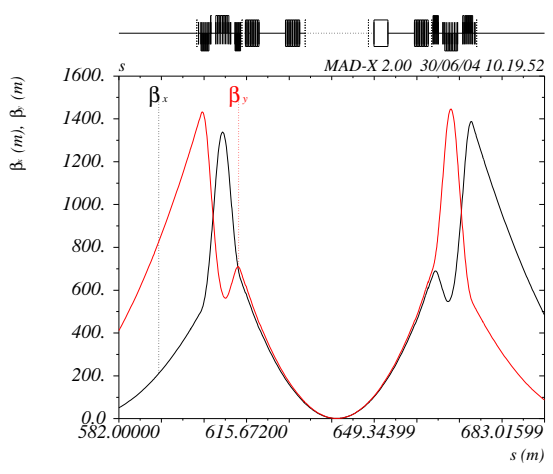

Figure 1: The optics and magnet locations in the 8 o'clock interaction region of the RHIC Blue ring.

\section{COMPARISON}

In this section we compare the corrector strengths from the action-angle kick minimization and the IR bump correction simulation. The IR bump correction strengths are calculated to minimize the residual linear or quadratic terms of the tune shifts to zero with two correctors. The beam is bumped only in the $8 \mathrm{o}$ 'clock interaction region of the RHIC Blue ring. And when we simulate the sextupole or octupole correction, only the sextupole or octupole field error are introduced into the interaction region. Other orders of the nonlinear errors are excluded to ensure the exact 


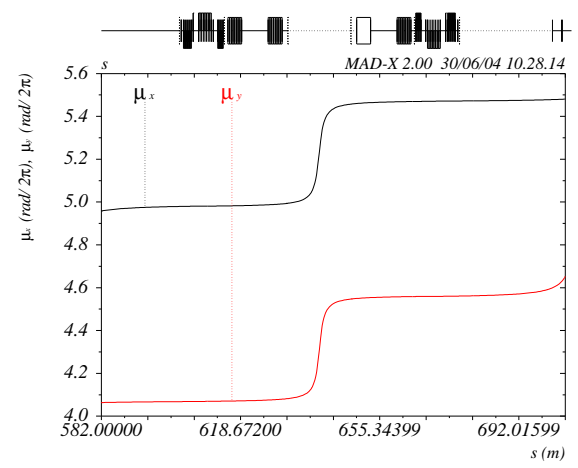

Figure 2: Betatron phase across 8 o'clock interaction region of the RHIC Blue ring.

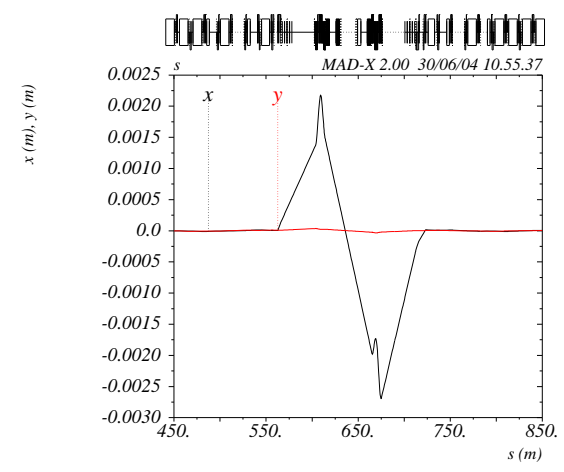

Figure 3: The orbits with the horizontal bump in the 8 o'clock interaction region of the RHIC Blue ring. All IR nonlinear errors are included.

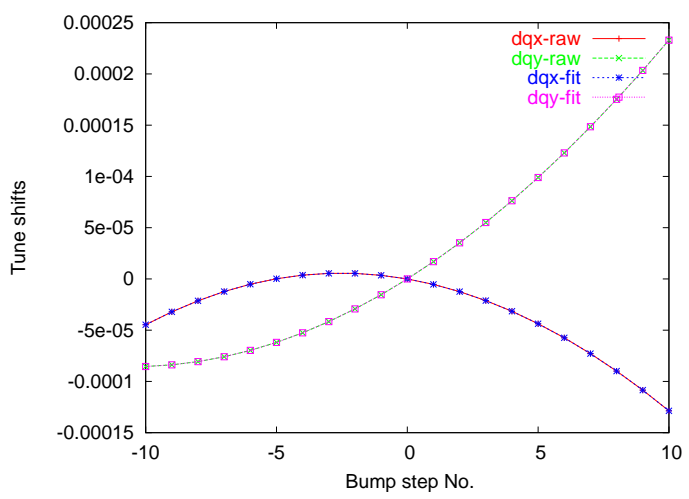

Figure 4: The tune shifts and the polynomial fitting curves.

comparisons of the right order field error correction.

In the following IR bump correction simulation, we use two sextupole or octupole correctors to minimize the linear terms of the $x$ and $y$ tune shifts to zero simultaneously. So we first need to obtain the contributions from the two correctors to the linear or quadratic terms. With the same IR bump, we get the tune shifts with one corrector on and off. The differences between them are due to the corrector. Through the polynomial fitting of the tune shift differences, we obtain the corrector's contribution to the linear

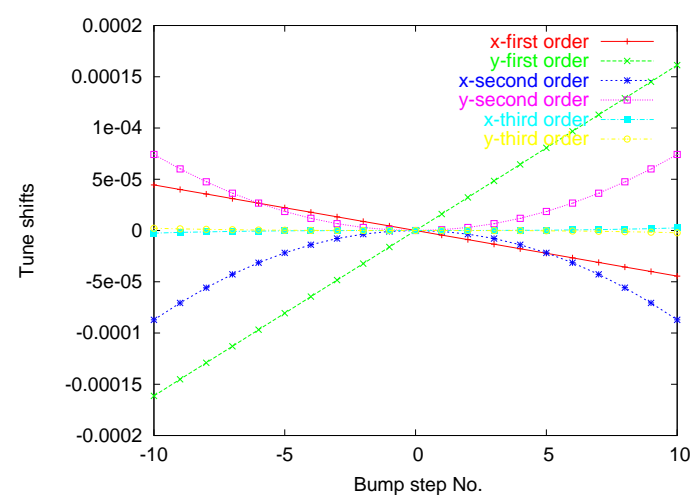

Figure 5: The tune shifts from the first three orders of polynomial fittings.

or quadratic terms of the tune shifts with a certain corrector's strength. Together with the residula linear or quadratic terms, the corrector strengths could be solved out by minimizing the $x$ and $y$ linear or quadratic terms to zero simultaneously.

\section{Sextupole Correction}

We use two sextupole correctors bo7-sx3 and bi8-sx3 for the sextupole field error correction in the Blue IR8. The three dipole kickers bo7-th4, bi8-th3 and bi8-th5 bump the beam in the $\mathrm{x}$ direction in 21 bump steps.

Table 2 gives the linear terms from the polynomial fittings of the tune shifts. The second block gives the residual linear terms from the sextupole field errors in the IR8. The third block gives the two correctors' contributions to the linear terms. With the two correctors' contribution to the linear terms of the tune shifts, the corrector strengths from the IR bump correction are solved out to minimize the $x$ and $y$ linear terms to zero simultaneously. The fourth block shows the linear terms of the tune shifts after setting back the corrector strengths from the off-line IR bump correction and the action-angle kick minimization. The corrector strengths from the action-angle kick minimization are calculated from the Eq. 1.

From the IR bump correction, the integrated corrector strengths for bo7-sx3 and bi8-sx3 are $-4.54 \times 10^{-3} \mathrm{~m}^{-2}$ and $2.74 \times 10^{-3} \mathrm{~m}^{-2}$, respectively. While from the actionangle kick minimization, the integrated corrector strengths for bo 7 -sx 3 and bi8-sx 3 are $-3.99 \times 10^{-3} \mathrm{~m}^{-2}$ and $2.97 \times$ $10^{-3} \mathrm{~m}^{-2}$, respectively. There is about a $10 \%$ difference in the correction strength of bo7-sx3. From Table 2, the linear terms of the tune shifts after the correction given by IR correction and the action-angle kick minimization are comparable. Figure 6 shows the tune shifts after the two corrections.

\section{Octupole Correction}

We use the two octupole correctors bo7-oct 2 and bi8oct 2 for the octupole filed error correction in the IR8. The same horizontal IR bump for the sextupole correction are 
Table 2: Sextupole correction for IR8 of RHIC Blue ring

\begin{tabular}{|c|c|c|}
\hline Conditions & Plane & $\begin{array}{l}\text { Linear term } \\
\text { Coefficient }\end{array}$ \\
\hline \multirow{2}{*}{$\begin{array}{l}\text { only } b_{2} \\
\text { errors }\end{array}$} & $\mathrm{X}$ & $\overline{7} 10.08 \times 10^{-5}$ \\
\hline & $\mathrm{y}$ & $-9.26 \times 10^{-5}$ \\
\hline \multirow{2}{*}{$\begin{array}{c}\text { bo7-sx3 } \\
k_{2} l=0.001 \mathrm{~m}^{-2}\end{array}$} & $\mathrm{X}$ & $5.65 \times 10^{-6}$ \\
\hline & $\mathrm{y}$ & $-1.43 \times 10^{-5}$ \\
\hline \multirow{2}{*}{$\begin{array}{c}\text { bi8-sx3 } \\
k_{2} l=0.001 \mathrm{~m}^{-2}\end{array}$} & $\mathrm{X}$ & $-2.74 \times 10^{-5}$ \\
\hline & $\mathrm{y}$ & $1.01 \times 10^{-5}$ \\
\hline \multirow{2}{*}{$\begin{array}{l}\text { IR bump } \\
\text { correction }\end{array}$} & $\bar{x}$ & $2.01 \times 10^{-6}$ \\
\hline & $\mathrm{y}$ & $-1.99 \times 10^{-6}$ \\
\hline \multirow{2}{*}{$\begin{array}{l}\text { Action-angle } \\
\text { correction }\end{array}$} & $\mathrm{X}$ & $-1.47 \times 10^{-6}$ \\
\hline & $\mathrm{y}$ & $-7.30 \times 10^{-6}$ \\
\hline
\end{tabular}

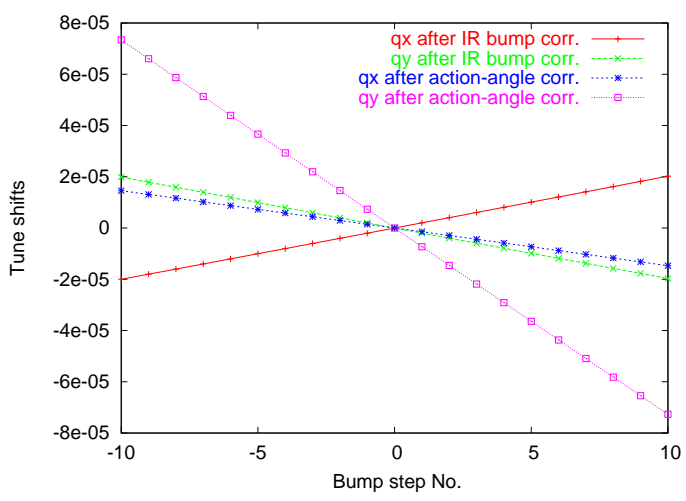

Figure 6: The tune shifts after the sextupole error corrections by the IR bump correction and the action-angle kick minimization.

used. Table 3 gives the tune shift quadratic terms from the polynomial fittings. From the IR bump correction, the in-

Table 3: Octupole correction for IR8 of RHIC Blue ring

\begin{tabular}{|c|c|c|}
\hline Conditions & Plane & $\begin{array}{c}\text { Quaratic Term } \\
\text { coefficient }\end{array}$ \\
\hline \multirow{2}{*}{$\begin{array}{c}\text { only } b_{3} \\
\text { errors }\end{array}$} & $\mathrm{X}$ & $-1.68 \times 10^{-7}$ \\
\hline & $\mathrm{y}$ & $6.40 \times 10^{-8}$ \\
\hline \multirow{2}{*}{$\begin{array}{c}\text { bo7-oct2 } \\
k_{3} l=0.001 \mathrm{~m}^{-3}\end{array}$} & $X$ & $1.58 \times 10^{-7}$ \\
\hline & $\mathrm{y}$ & $-8.74 \times 10^{-8}$ \\
\hline \multirow{2}{*}{$\begin{array}{c}\text { bi8-oct2 } \\
k_{3} l=0.001 \mathrm{~m}^{-3}\end{array}$} & X & $7.94 \times 10^{-8}$ \\
\hline & $\mathrm{y}$ & $-1.43 \times 10^{-7}$ \\
\hline \multirow{2}{*}{$\begin{array}{c}\text { IR bump } \\
\text { correction }\end{array}$} & $\mathrm{X}$ & $-5.61 \times 10^{-9}$ \\
\hline & $\mathrm{y}$ & $3.13 \times 10^{-9}$ \\
\hline \multirow{2}{*}{$\begin{array}{l}\text { Action-angle } \\
\text { correction }\end{array}$} & $\mathrm{x}$ & $-6.84 \times 10^{-8}$ \\
\hline & $\mathrm{y}$ & $3.08 \times 10^{-8}$ \\
\hline
\end{tabular}

tegrated corrector strengths for bo7-oct 2 and bi8-oct 2 are $0.121 \mathrm{~m}^{-3}$ and $-0.029 \mathrm{~m}^{-3}$, respectively. While from the action-angle kick minimization, the integrated correction strengths for bo 7 -sx 3 and bi8-sx 3 are $0.0768 \mathrm{~m}^{-3}$ and $-0.023 \mathrm{~m}^{-3}$, respectively. There are about $30 \%$ differ- ence in the corrector strengths of bo7-oct2. From Table 2, the quadratic terms of the tune shifts with the IR bump correction strengths is smaller than these with the action-angle kick minimization correction strengths. Figure 7 shows the contributions of the quadratic terms of the tune shifts after the corrections.

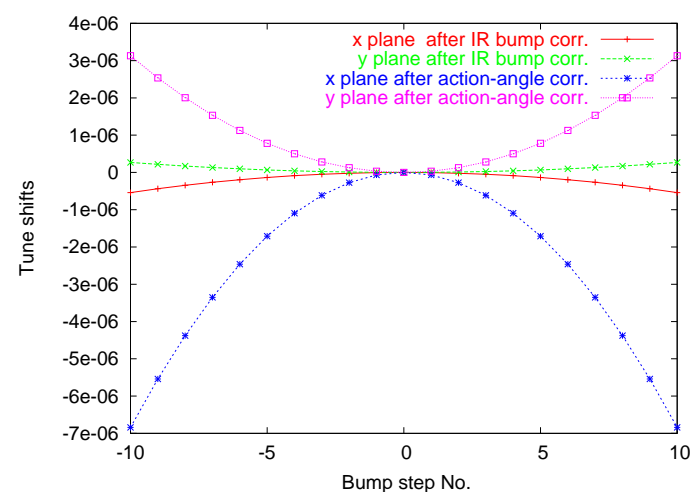

Figure 7: The quadratic term tune shift contributions after octupole error corrections by IR bump correction and action-angle kick minimization.

\section{ANALYSIS}

From the off-line IR bump correction and the action angle minimization, we found that there are about $10 \%$ difference for the sextupole corrector strengths, and about $30 \%$ difference for the octupole corrector strengths. However, from the above analytical solutions to the IR bump correction for the sextupole, the two methods are identical. In the following, we will check item by item to find out where the problem comes from. First we will check the tune shifts from one sextupole or one octupole, then check the tune shifts from two sextupoles or two octupoles.

\section{Bump Amplitudes and Error Strengths}

Here we check the tune shift due to one sextupole or one octupole from the off-line IR-bump. In our simulation, we only switch on one sextupole or one octupole corrector each time.

From Eq.6, the tune shifts from the horizontal orbit in one sextupole should be proportional to the bump amplitude. The linear term of the tune shift polynomial fitting should be proportional to the sextupole's integrated strength. Figure 8 shows the tune shifts from the off-line IR bump with the linearly increased bump amplitudes. Figure 9 shows the linear term versus the sextupole integrate strengths. Both agree well with the predications.

From Eq.11, the tune shifts from the horizontal orbit in one octupole should be proportional to the bump amplitude. The quadratic terms of the tune shift polynomial fitting should be proportional to the octupole's integrated strengths. Figure 10 shows the tune shifts from the offline IR bump with the linearly increased bump amplitudes. 
Figure 11 shows the quadratic terms versus the octupole integrate strengths. Both agree the predications well, too.

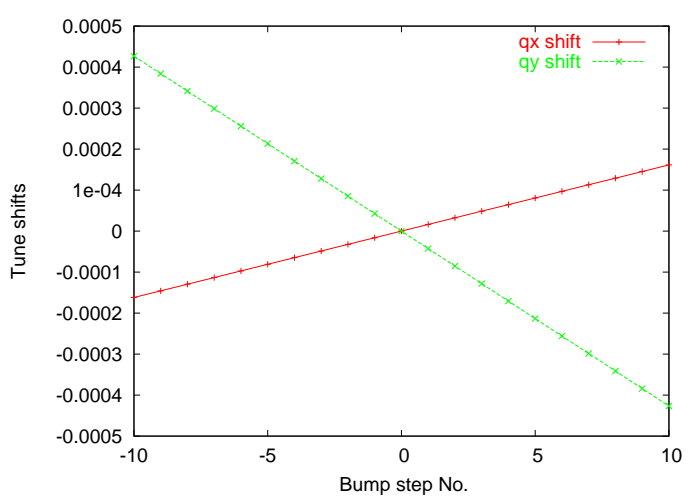

Figure 8: The tune shifts versus the bump amplitudes due to one sextupole.

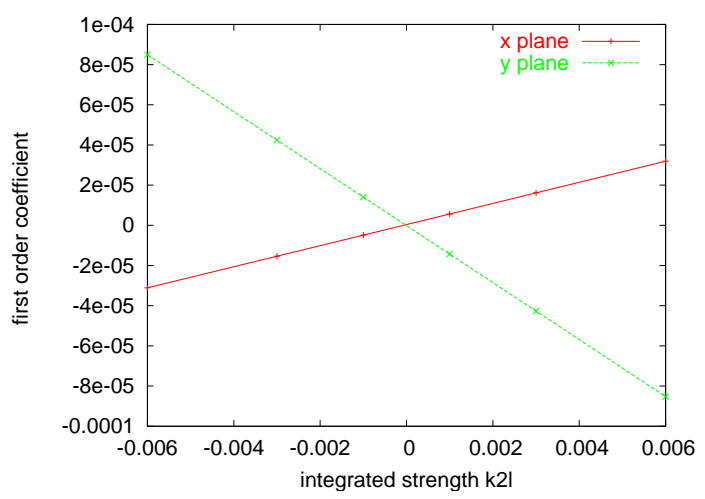

Figure 9: The linear term of tune shifts versus the integrated sextupole strengths.

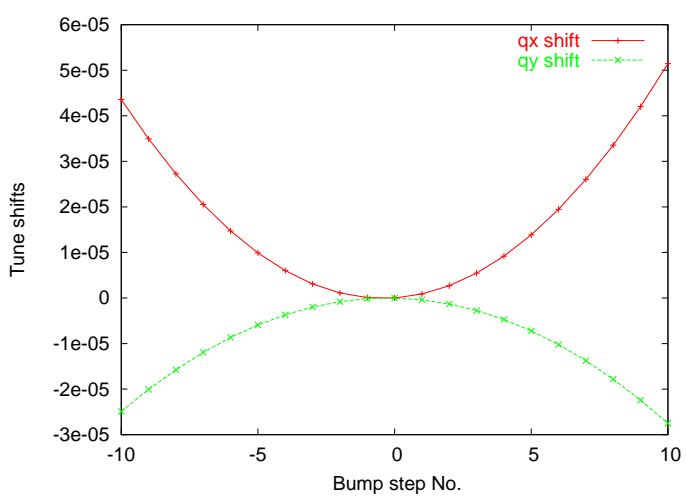

Figure 10: The tune shifts versus the bump amplitudes due to one octupole.

\section{Tune Shifts in $x / y$ Planes}

We check here the tune shift ratios of the horizontal and the vertical planes only from one sextupole or one octupole. Table 4 gives the optical functions at the dipole kickers, the sextupole and the octupole correctors used in this note.

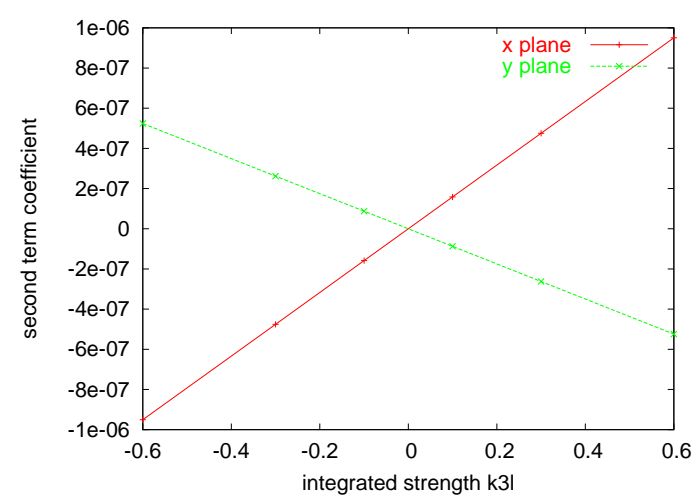

Figure 11: The quadratic term of tune shifts versus the integrated octupole strengths.

Table 4: Optics parameters for IR8 of RHIC Blue ring

\begin{tabular}{|c|c|c|c|}
\hline Element & $\begin{array}{c}\mathrm{s} \\
\text { (in } \mathrm{m} \text { ) }\end{array}$ & $\begin{array}{c}\beta_{x} / \beta_{y} \\
\text { ( in m ) }\end{array}$ & $\begin{array}{c}\text { mux/muy } \\
\text { ( in } 2 \pi \text { ) }\end{array}$ \\
\hline \hline bo7-th4 & 562.78 & $28.50 / 43.28$ & $4.53 / 4.03$ \\
\hline bi8-th3 & 675.87 & $1297.89 / 477.16$ & $5.48 / 4.55$ \\
\hline bi8-th5 & 723.24 & $76.97 / 15.11$ & $5.54 / 5.07$ \\
\hline bo7-oct2 & 611.46 & $1042.11 / 574.92$ & $4.99 / 4.06$ \\
\hline bo7-oct3 & 603.02 & $479.03 / 1292.72$ & $4.99 / 4.06$ \\
\hline bi8-oct2 & 667.43 & $577.37 / 1037.87$ & $5.48 / 4.55$ \\
\hline bi8-oct3 & 675.87 & $1297.89 / 477.16$ & $5.48 / 4.55$ \\
\hline bo7-sx3 & 603.02 & $479.03 / 1292.72$ & $4.99 / 4.06$ \\
\hline bi8-sx3 & 675.87 & $1297.89 / 477.16$ & $5.48 / 4.55$ \\
\hline
\end{tabular}

From Eq. 6 and Eq. 11 , the ratios are only determined by the $\beta_{x}$ and $\beta_{y}$. For one sextupole or one octupole, the ratio of the linear and quadratic $x$ and $y$ tune shifts equals $\left(\beta_{x}: \beta_{y}\right)$.

If there is only sextupole corrector bo7-sx3 on in the interaction region, whose integrated strength is $0.01 \mathrm{~m}^{-2}$. we get the linear term coefficient from the horizontal and vertical tunes $5.29 \times 10^{-5}$ and $-14.19 \times 10^{-5}$, respectively. Their ratio is about $1:(-2.68)$. The $\beta_{x}: \beta_{y}$ at bo7-sx3 is $479.03: 1292.72=1: 2.69$. The opposite signs of the tune shifts can be explained from Eq. 1. So we say the simulation result agrees well with the prediction.

If there is only one octupole corrector bo7-oct 2 on in the interaction region, whose integrated strength is $0.1 \mathrm{~m}^{-3}$. we get the quadratic terms from the horizontal and vertical tunes $1.58 \times 10^{-7}$ and $-8.74 \times 10^{-8}$, respectively. Their ratio of them is $1.81:(-1)$. The $\beta_{x}: \beta_{y}$ at bo7-oct2 is $1042: 574.92=1.81: 1$. The opposite signs of the tune shifts can be explained from Eq. 1. So we say the simulation result agrees with the analytical predication, too.

\section{Two Sextupoles or Octupoles}

Here we check whether the polynomial fitting terms could be added or not for two sextupoles or two octupoles.

We first switch on the two sextupoles or octupoles one by 
one, and get their contributions to the linear or quadratic terms. Then switch on both of them. Table 5 shows the first and quadratic terms for two sextupole and octupole correctors. The integrated strengths of the two sextupole correctors bo7-sx3, bi8-sx3 both are $0.01 \mathrm{~m}^{-2}$. The integrated strengths of the two octtupole correctors bo7-oct2, bi8-oct 2 both are $0.1 \mathrm{~m}^{-3}$. According to Table 5, we can see the contributions from different individual sextupoles and octupoles are addable.

Table 5: Tune shift polynomial terms from two correctors

\begin{tabular}{|c|c|c|}
\hline Correctors & x plane & y plane \\
\hline \hline bo7-sx3 & $5.29 \times 10^{-5}$ & $14.19 \times 10^{-5}$ \\
\hline bi8-sx3 & $-27.79 \times 10^{-5}$ & $10.22 \times 10^{-5}$ \\
\hline Above Two & $-22.54 \times 10^{-5}$ & $-3.96 \times 10^{-5}$ \\
\hline \hline bo7-oct2 & $1.58 \times 10^{-7}$ & $-8.74 \times 10^{-8}$ \\
\hline bi8-oct2 & $7.94 \times 10^{-8}$ & $-1.43 \times 10^{-8}$ \\
\hline Above Two & $2.38 \times 10^{-7}$ & $-2.30 \times 10^{-7}$ \\
\hline
\end{tabular}

\section{Contribution Ratios}

Now we turn to check the ratios of the polynomial terms from two individual sextupole's or octupoles. From Eq. 10, it should be proportional to $\beta^{3 / 2}$ for two sextupoles with the same integrated strength. In the above example, the ratio of the linear term of the horizontal tune shifts from two sextupoles bo7-sx3 and bi8-sx3 with the same integrated strength $0.01 \mathrm{~m}^{-2}$. is :

$$
\begin{aligned}
& \left.\Delta \nu_{x}\right|_{b o 7-s x 3}:\left.\Delta \nu_{x}\right|_{b i 8-s x 3} \\
& =5.29 \times 10^{-5}: 27.79 \times 10^{-5} \\
& =1: 5.25
\end{aligned}
$$

The ratio of the $\beta^{3 / 2}$ of the two sextupoles are:

$$
\begin{aligned}
& \left.\beta_{x}^{3 / 2}\right|_{b o 7-s x 3}:\left.\beta_{x}^{3 / 2}\right|_{b i 8-s x 3} \\
& =479.03^{3 / 2}: 1297.89^{3 / 2} \\
& =1: 4.46
\end{aligned}
$$

so from our IR bump simulation, the ratio of the linear tune shift terms is not proportional to the ratio of $\beta^{3 / 2}$ for two sextupoles with the same integrated strength. This only reason for the unequality is :

$$
\begin{aligned}
& \left.x_{c o}\right|_{b o 7-s x 3}:\left.x_{c o}\right|_{b i 8-s x 3} \\
& \neq\left.\beta_{x}^{1 / 2}\right|_{b o 7-s x 3}:\left.\beta_{x}^{1 / 2}\right|_{b i 8-s x 3} .
\end{aligned}
$$

This guess is verified by the following orbit bump check at these two sextupole correctors. From the IR bump simulation with MADX,

$$
\begin{gathered}
\left.x_{c o}\right|_{b o 7-s x 3}:\left.x_{c o}\right|_{b i 8-s x 3} \\
=1: 1.955 \\
\left.\beta_{x}^{1 / 2}\right|_{b o 7-s x 3}:\left.\beta_{x}^{1 / 2}\right|_{b i 8-s x 3} \\
=1: 1.646
\end{gathered}
$$

Substituting the real orbit ratio of $x_{c o}$ instead of the ratio of $\beta_{x}^{1 / 2}$ into Eq. 6, we get the horizontal tune shift contribution ratio from the two sextupoles:

$$
\begin{gathered}
\left.\left(x_{c o} \beta_{x}\right)\right|_{b o 7-s x 3}:\left.\left(x_{c o} \beta_{x}\right)\right|_{b i 8-s x 3} \\
=1: 5.30
\end{gathered}
$$

which is much closer to the linear term tune shift ratio we get from the IR bump simulation.

Same as the sextupole check, we check the quadratic term tune shift ratios of two individual octupoles bo7-oct2 and bi8-oct 2 with the same integrated strength $0.1 \mathrm{~m}^{-3}$. From the simulation, we get :

$$
\begin{aligned}
& \left.\Delta \nu_{x}\right|_{\text {bo } 7-o c t 2}:\left.\Delta \nu_{x}\right|_{\text {bi8-oct } 2} \\
& =1.58 \times 10^{-7}: 7.94 \times 10^{-8} \\
& =1.996: 1
\end{aligned}
$$

However,

$$
\begin{aligned}
& \left.\beta_{x}^{2}\right|_{b o 7-s x 3}:\left.\beta_{x}^{2}\right|_{b i 8-s x 3} \\
& =1042.11^{2}: 577.37^{2} \\
& =3.261: 1
\end{aligned}
$$

Here the ratio of the tune shifts are not equal to the ratio of $\beta_{x}^{2}$ as assumed from Table 1, either. The ratio of orbit amplitudes at the two octupoles is:

$$
\begin{gathered}
\left.x_{c o}\right|_{b o 7-o c t 2}:\left.x_{c o}\right|_{b i 8-o c t} 2 \\
=1.051: 1
\end{gathered}
$$

Substituting $x_{c o}$ ratio instead of $\beta_{x}^{1 / 2}$ ratio into Eq. 11 , we obtain the horizontal tune shift contribution ratio from the two octupoles:

$$
\begin{gathered}
\left.\left(x_{c o}^{2} \beta_{x}\right)\right|_{b o 7-s x 3}:\left.\left(x_{c o}^{2} \beta_{x}\right)\right|_{b i 8-s x 3} \\
=1.995: 1
\end{gathered}
$$

which is almost the same as that from the IR bump simulation.

\section{COMMENTS}

After the above step-by-step check, we finally find the reason for the differences of the correction strengths from the IR bump correction and the action-angle kick minimization. Since in the IR bump, the horizontal orbit is not exactly proportional to the $\beta_{x}^{1 / 2}$, the equations for actionangle kick minimization Eq. 10, Eq. 13 are not exactly simulated by the IR bump correction method.

The source for this difference is that the phase advance over the interaction region is not exactly equal to $\pi$. Presenting the phase advance as $\Delta \Psi=\pi+\Delta \psi$ and using Eq. 14 one can get the ratio of orbit positions on the left and right sides of the interaction region as

$$
\begin{aligned}
\frac{x_{c o, L}}{x_{c o, R}} & =\frac{\sqrt{\beta_{x, L}} \sin \left(\Psi_{0}\right)}{\sqrt{\beta_{x, R}} \sin \left(\Psi_{0}+\Delta \Psi\right)} \\
& \simeq-\frac{\sqrt{\beta_{x, L}}}{\sqrt{\beta_{x, R}}}\left(1-\cot \left(\Psi_{0}\right) \Delta \psi\right)
\end{aligned}
$$


Although $\Delta \psi$ is small the $\cot \psi_{0}$ can reache 15 units for RHIC IR bump, which leads to considerable difference between the ratio of the orbits and the ratio of $\sqrt{\beta}$. Then the correction strengths from the IR bump correction are not the same as that from the action-angle kick minimization analytical prediction.

The action-angle assumes the betatron phase advance is exactly $\pi$. If we don't ignore the phase in the action-angle kick minimization, Eq. 10 and Eq. 13 for the sextupole correction and octupole correction should be :

$$
\left\{\begin{array}{ll}
e^{i\left(n \Psi_{x}\right)}, & \text { in the X-plane } \\
e^{i\left[\Psi_{x}+(n-1) \Psi_{y}\right]} \text { or } e^{i\left(n \Psi_{y}\right)} & \text { in the y-plane }
\end{array} .\right.
$$

So the two methods, IR bump correction and the actionangle kick minimization, have different approximations in the betatron phase advance. Limited by the IR bump amplitudes and the tune measurement resolution, the IR bump correction method is more suited to the lower order nonlinear field error corrections. The action-angle kick minimization could be used to high nonlinear order correction. However, the IR bump correction is operational, while the action-angle kick minimization only works off-line for known nonlinear error correction.

\section{FURTHER DISCUSSION OF IR BUMP}

In this section, we will make further discussions about the usages of the IR bump nonlinear correction method.

\section{Octupole Correction}

As we have mentioned above that the weight factor in the vertical plane for the octupole error correction using the horizontal bump is different from that from the action-angle kick minimization. According to Table 1, the equations for the octupole error correction should be:

$$
\left\{\begin{array}{l}
\sum_{L} b_{3} d s \beta_{x}^{2}+\sum_{R} b_{3} d s \beta_{x}^{2}=0 \\
\sum_{L} b_{3} d s \beta_{y}^{2}+\sum_{R} b_{3} d s \beta_{y}^{2}=0
\end{array},\right.
$$

which is different from Eq. 13 from the horizontal IR bump correction.

From the Hamiltonian perturbation theory, the tune shifts from the orbits in the octupoles are given:

$$
\left\{\begin{array}{l}
\Delta Q_{x}=\frac{1}{2 \pi} \oint\left(12 b_{3} x_{c o}^{2} \beta_{x}-12 b_{3} y_{c o}^{2} \beta_{x}\right) d s \\
\Delta Q_{y}=\frac{1}{2 \pi} \oint\left(-12 b_{3} x_{c o}^{2} \beta_{y}+12 b_{3} y_{c o}^{2} \beta_{y}\right) d s
\end{array}\right.
$$

So one solution is to minimize the horizontal quadratic term tune shift in the horizontal IR bump and the vertical quadratic term tune shift in the vertical IR bump simultaneously, while ignoring the vertical quadratic term tune shift in the horizontal IR bump and the horizontal quadratic tune shift in the vertical IR bump. The vertical dipole kickers bo7-tv5, bo7-tv3, bi8-tv4 are used to produce the vertical IR bump across IR8 in the simulation.

Table 6 shows the exact correction procedure. The numbers in red color are the quadratic terms of the horizontal tune shifts in the horizontal IR bump, the numbers in blue color are the quadratic terms of the vertical tune shifts in the vertical IR bump. The correction strengths are solved out from these colored numbers. The correction strengths for bo7-oct2 and bi8-oct2 are $0.14782 \mathrm{~m}^{-3}$ and -0.0831 $\mathrm{m}^{-3}$, respectively.

Table 6: Octupole correction for IR8 of RHIC Blue ring

\begin{tabular}{|c|c|c|c|}
\hline Case & $\begin{array}{c}\text { tune } \\
\text { plane }\end{array}$ & $\begin{array}{c}\text { Horizontal } \\
\text { IR bump }\end{array}$ & $\begin{array}{c}\text { Vertical } \\
\text { IR bump }\end{array}$ \\
\hline \hline \multirow{2}{*}{$\begin{array}{c}\text { only } b_{3} \\
\text { errors }\end{array}$} & $\mathrm{x}$ & $-1.68 \times 10^{-7}$ & $6.97 \times 10^{-8}$ \\
\cline { 2 - 4 } & $\mathrm{y}$ & $6.40 \times 10^{-7}$ & $3.30 \times 10^{-8}$ \\
\hline \hline bo7-oct2 & $\mathrm{x}$ & $1.58 \times 10^{-7}$ & $-3.25 \times 10^{-7}$ \\
\cline { 2 - 4 } K2L=0.1 & $\mathrm{y}$ & $-8.74 \times 10^{-8}$ & $1.79 \times 10^{-7}$ \\
\hline bi8-oct2 & $\mathrm{x}$ & $7.94 \times 10^{-7}$ & $-1.99 \times 10^{-7}$ \\
K2L=0.1 & $\mathrm{y}$ & $-1.43 \times 10^{-8}$ & $3.58 \times 10^{-7}$ \\
\hline \hline \multirow{2}{\text{after}}{} & $\mathrm{x}$ & $-6.11 \times 10^{-9}$ & \\
\cline { 2 - 4 } correction & $\mathrm{x}$ & & $-3.11 \times 10^{-9}$ \\
\hline
\end{tabular}

However, the correction procedure is much complicated since we have to perform two planes IR bumps. One simple alternative is what we did above only with the horizontal IR bump. The weight factors are $\beta_{x}^{2}, \beta_{x} \beta_{y}$ for the only horizontal bump, instead of the $\beta_{x}^{2}, \beta_{y}^{2}$ for the two plane bumps. For the above case, if we only use the horizontal IR bump as shown before, the corrector strengths for the bo7-oct2 and bi8-oct 2 are $0.121 \mathrm{~m}^{-3}$ and $-0.029 \mathrm{~m}^{-3}$, respectively. After the correction, the quadratic term of the horizontal tune shift polynomial fitting from the horizontal IR bump is $-5.61 \times 10^{-9}$, and the quadratic term of the vertical tune shift from the vertical IR bump is $3.13 \times 10^{-9}$. Comparing to those numbers from Table 6 , the correction is satisfactory.

For the corrections of all the resonance induced by the octupole fields in Eq. 5, there are three kinds of the weight factors, $\beta_{x}^{2}, \beta_{x}^{2}$ and $\beta_{x} \beta_{y}$. To fully correct all the resonance strengths from the octupoles, both horizontal and vertical IR bumps are needed. The total four quaratic terms of the $x$ and $y$ tune shifts from the horizontal and vertical IR bumps should be minimized simultaneously. The existing 4 octupole correctors in each interaction region should be enough to realize this correction on line.

In fact, there are flexibilities to choose the weight factors. It really depends on what formalism to use to obtain them. LHC uses different weight factors for the high order $b_{6}$ and $b_{10}$ correction [8]. It shows that there are no big difference in the correction strengths between the two sets of the weight factors for the $b_{6}$ and $b_{10}$ corrections.

\section{Skew Sextupole Correction}

From the Hamiltonian perturbation theory, the tune shifts due to the vertical orbit offset in the skew sextupoles are 
given:

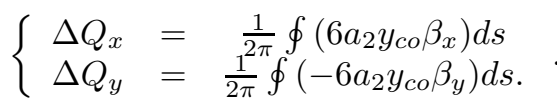

So we could perform its correction through the vertical IR bump and minimizing the linear horizontal and vertical tune shift polynomial coefficients with two individual skew sextupole correctors. The two equations for the minimization are:

$$
\left\{\begin{array}{ccc}
\sum_{L} a_{2} d s \beta_{x} \beta_{y}^{1 / 2}-\sum_{R} a_{2} d s \beta_{x} \beta_{y}^{1 / 2} & = & 0 \\
\sum_{L} a_{2} d s \beta_{y}^{3 / 2}-\sum_{R} a_{2} d s \beta_{y}^{3 / 2} & = & 0
\end{array},\right.
$$

which is the same as that from Table 1.

The vertical dipole kickers bo7-tv5, bo7-tv3, bi8-tv4 are used to produce the vertical IR bump across IR8 in the simulation. Figure 12 and Table 7 show the tune shifts and the linear term polynomial coefficients before and after skew quadrupole corrections. The two skew sextupole correctors are bo7-sxs 3 and bi8-sxs3. Their integrated correction strengths from simulation are $0.000217 \mathrm{~m}^{-2}$ and -0.000354 $\mathrm{m}^{-2}$, respectively.

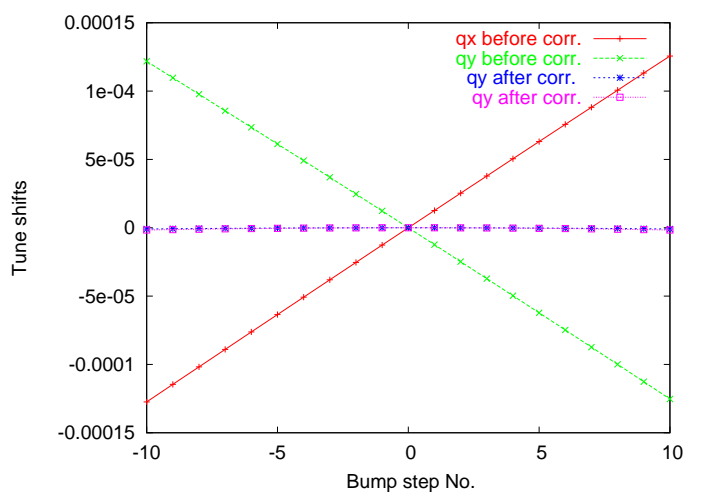

Figure 12: Tune shifts before and after the off-line skew sextupole correction.

Table 7: Skew sextupole correction from off-line IR bump

\begin{tabular}{|c|c|c|}
\hline Conditions & plane & Linear Term Coeff. \\
\hline \hline only b3 & $\mathrm{x}$ & $1.26 \times 10^{-7}$ \\
\cline { 2 - 3 } errors in IRs & $\mathrm{y}$ & $-1.23 \times 10^{-7}$ \\
\hline after b3 & $\mathrm{x}$ & $-2.19 \times 10^{-10}$ \\
\cline { 2 - 3 } correction & $\mathrm{y}$ & $-7.91 \times 10^{-10}$ \\
\hline
\end{tabular}

\section{Skew Octupole Correction}

The tune shifts due to the orbit offset in the skew octupoles are given:

$$
\left\{\begin{array}{l}
\Delta Q_{x}=\frac{1}{2 \pi} \oint\left(6 a_{3} x_{c o} y_{c o} \beta_{x}\right) d s \\
\Delta Q_{y}=\frac{1}{2 \pi} \oint\left(-6 a_{3} x_{c o} y_{c o} \beta_{y}\right) d s
\end{array} .\right.
$$

So one possible correction method is to bump the beam in the horizontal and vertical planes simultaneously, and to to minimize the quadratic term coefficients of the horizontal and vertical tune shifts with two individual skew octupole correctors. The two equations for the minimization are:

$$
\left\{\begin{array}{c}
\sum_{L} a_{3} d s \beta_{x}^{3 / 2} \beta_{y}^{1 / 2}+\sum_{R} a_{3} d s \beta_{x}^{3 / 2} \beta_{y}^{1 / 2}=0 \\
\sum_{L} a_{3} d s \beta_{x}^{1 / 2} \beta_{y}^{3 / 2}+\sum_{R} a_{3} d s \beta_{x}^{1 / 2} \beta_{y}^{3 / 2}=0
\end{array},\right.
$$

which is the same as that from Table 1.

The horizontal dipole kickers bo7-th4, bi8-th3, bi8-th5 and the vertical dipole kickers bo7-tv5, bo7-tv3, bi8-tv4 are used here to produce the wanted horizontal and vertical IR bumps across IR8. Figure 13 shows the IR bumps from MADX. Table 8 and Figure 14 shows the tune shifts before and after skew quadrupole corrections. The two skew octupole correctors are bo7-octs3 and bi8-octs3. Their integrated correction strengths from the off-line IR bump are $0.0046 \mathrm{~m}^{-3}$ and $0.0021 \mathrm{~m}^{-3}$, respectively.

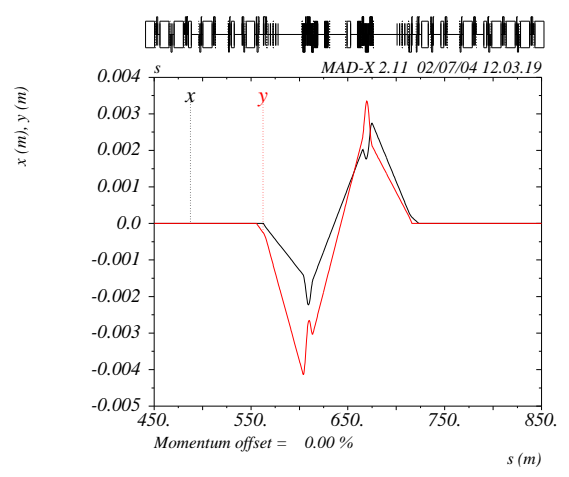

Figure 13: The IR bump for the off-line skew octupole correction.

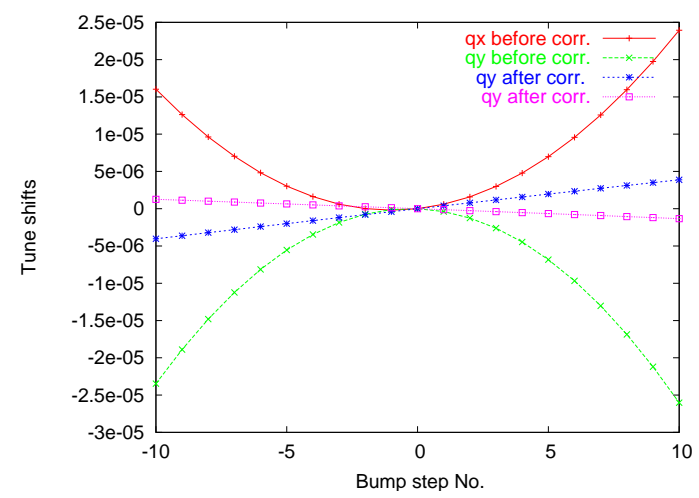

Figure 14: Tune shifts before and after the off-line skew octupole correction.

\section{CONCLUSION}

The correction strengths from the off-line IR bump correction method are compared to that from the action-angle kick minimization. It shows that there are some discrepancies. The reason was found after the step-by-step check. 
Table 8: Skew octupole correction from off-line IR bump

\begin{tabular}{|c|c|c|}
\hline Conditions & plane & Quadratic Term Coeff. \\
\hline \hline only b3 & $\mathrm{x}$ & $2.00 \times 10^{-7}$ \\
\cline { 2 - 3 } errors in IRs & $\mathrm{y}$ & $-2.47 \times 10^{-7}$ \\
\hline after b3 & $\mathrm{x}$ & $-6.39 \times 10^{-10}$ \\
\cline { 2 - 3 } correction & $\mathrm{y}$ & $-4.02 \times 10^{-10}$ \\
\hline
\end{tabular}

The not exact $\pi$ phase advance between the two sides of the interaction region makes that the bump orbit not exacly proportionaly to the $\beta^{1 / 2}$. The action-angle kick minimization and the IR bump correction are not exacltly identical for the sextupole and octupole field error corrections. Comments to the octupole, skew sextupole, skew octupole field error corrections with IR bump correction method are given. The on-line procedures are suggested for more effective corrections of these nonlinear errors. The bump closure and the field feeddown effects in the real machines are not covered.

\section{ACKNOWLEDGEMENT}

The authors thank N. Malitsky for the simulating discussion on off-line IR correction simaultion and thank R. Tomás for supplying the RHIC MADX lattice with the IR nonlinear modeling.

\section{REFERENCES}

[1] J-P. Koutchouk, F. Pilat, V. Ptitsyn, et al, PAC2001.

[2] F. Pilat, V. Ptitsyn, EPAC2002, Paris, France.

[3] J. Wei, Proceedings of the Workshop on LHC Interaction Region Correction Systems, BNL, May 6-7, 1999.

[4] R. Tomás, et al., EPAC2004, Luzen, Switzerland.

[5] Proceedings of the Workshop on LHC Interaction Region Correction Systems, BNL, May 6-7, 1999.

[6] H. Wiedemann, Particle Accelerator Physics: Nonlinear and Higher-order Beam Dynamics, Springer Press, 1994.

[7] Madx code, CERN, http://mad.home.cern.ch/mad/.

[8] H. Grote, et al., LHC-project-note 197. 